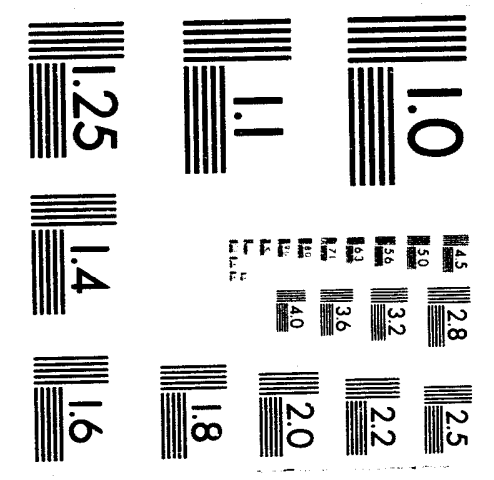



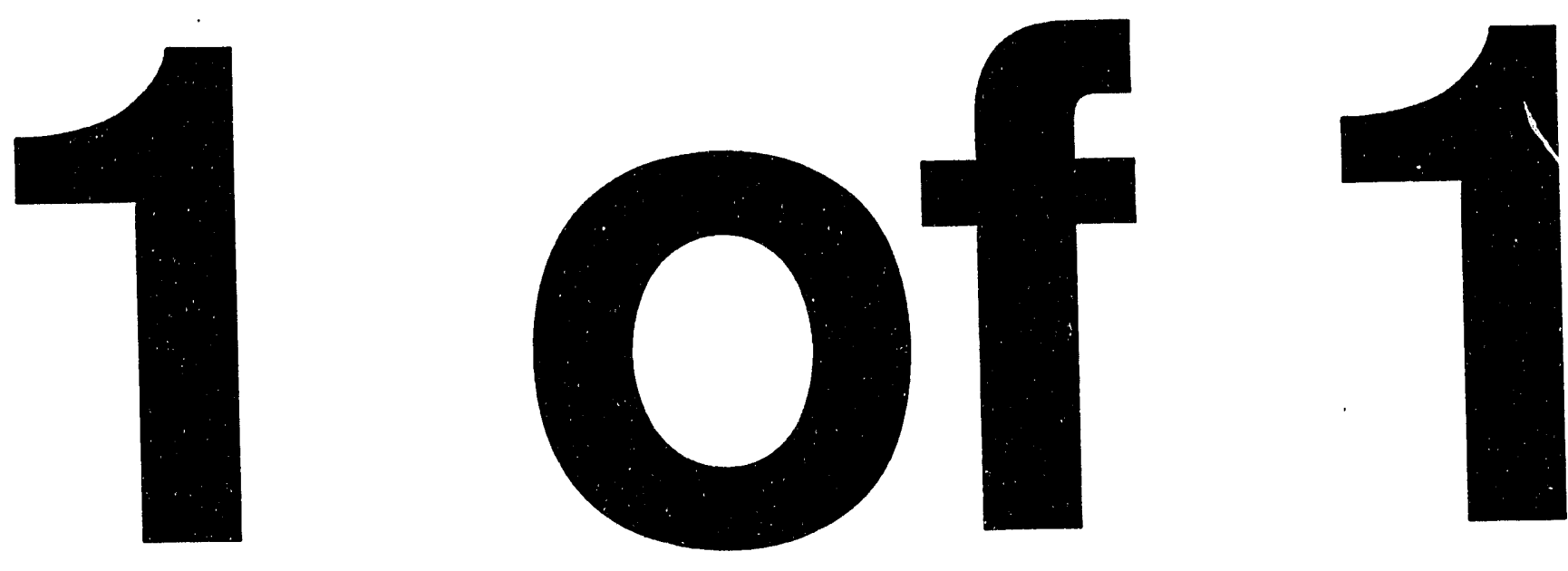


\section{INSTITUTE FOR RECEIVED FUSION STUDIES \\ APR 111994 \\ OSTI}

DE-FG05-80ET-53088-647

IFSR \#647

Dynamical Nature of Inviscid Power Law for

Two-Dimensional Turbulences and Self-Consistent

Spectrum and Transport of Plasma Filaments

Y.Z. ZHANG ${ }^{a}$ and S.M. MAHAJAN

Institute for Fusion Studies

The University of Texas at Austin

Austin, Texas 78712

February 1994

a) ICTP, Trieste, Italy

\section{THE UNIVERSITY OF TEXAS}

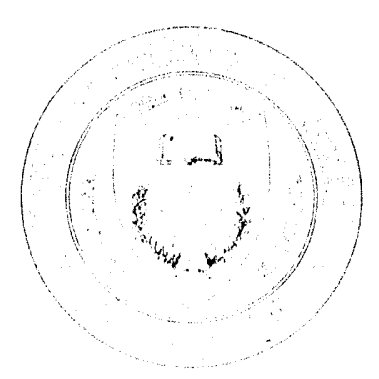

\section{AUSTIN}




\title{
Dynamical Nature of Inviscid Power Law for Two-Dimensional Turbulences and Self-Consistent Spectrum and Transport of Plasma Filaments
}

\author{
Y.Z. ZHANG ${ }^{a)}$ AND S.M. MAHAJAN \\ Institute for Fusion Studies \\ The University of Texas at Austin \\ Austin, Texas 78712
}

\begin{abstract}
On the basis of equal-time correlation theory (a non-perturbative approach) inviscid power laws of $2 \mathrm{D}$ isotropic plasma turbulences with one Lagrangian inviscid constant of motion are unambiguously solved by determining the dynamical characteristics. Two distinct types of induced transport, according to the divergence of the inverse correlation length in the inviscid limit, are revealed. This analysis also suggests a physically reasonable closure. The self-consistent system (a set of integral equations) for plasma filaments is investigated in detail, and is found to be a nonlinear differential eigenvalue problem for the diffusion coefficient $D$, with the Dyson-like (integral) equation playing the role of a boundary condition. This new type of transport is non-Bohm-like, and shows quasilinear behavior even in the strong turbulence regime. Physically, this behavior arises from a synchronization of the shrinking squared correlation length with the decorrelation time, for which the "mixing-length" breaks down. The shrinkage of correlation length is a characteristic pertaining to the new type of turbulence; its relationship with the turbulence observed in supershot regime on TFTR is commented on.
\end{abstract}

a) ICTP, Trieste, Italy 
It is generally believed that the Kolmogorov $-5 / 3$ inviscid power law for $3 \mathrm{D}$ incompressible fluids is a manifestation of the scale invariance of the conserved energy $\varepsilon .{ }^{1}$ However, for $2 \mathrm{D}$ incompressible fluids the squared vorticity $\Omega$ is also a constant of motion. The arguments based on the scale invariance of $\Omega$ lead to an alternative -3 inviscid power law, in contrast. to the one obtained by assuming $\varepsilon$ as a scale invariant quantity. ${ }^{2}$ The numerical simulations for the Hasegawa-Mima (HM), and Hasegawa-Wakatani (HW) equations ${ }^{3,4}$ seem to support the -3 power law, suggesting that the latter Lagrangian inviscid constant of motion, from which infinite constants of motion can be generated, would play a special role in determining the nature of turbulence spectrum in the inviscid range.

The Lagrangian inviscid constant of motion is a field $\Psi$, obeying the equation of incompressible $2 \mathrm{D}$ fluids

$$
\left(\frac{\partial}{\partial t}+\mathbf{z} \times \nabla \varphi \cdot \nabla-\xi \nabla^{2}\right) \Psi=0,
$$

where $\xi \nabla^{2}$ represents a model dissipation at short wavelengths ("inviscid" throughout this Letter refers solely to the vanishing limit of the short wavelength dissipation), $\varphi$ is an appropriate stream function, and $\mathbf{z}$ is the unit vector perpendicular to the 2D-plane. For example, the HM and the HW systems are respectively described by $\Psi \equiv \ln n_{0}+\varphi-\nabla^{2} \varphi$ and $\Psi \equiv \ln n-\nabla^{2} \varphi$. In either case $\varphi$ is the electrostatic potential, $n_{0}(n)$ is the equilibrium (total) plasma density. The continuity equation for convective cells also obeys Eq. (1). In this Letter we explore a similar model, the plasma filaments model (PF), which, except for dissipations, is very much like the trapped ion model..$^{5}$ The ion filaments in this model satisfy the equivalent of Eq. (1) in the form $d n_{i} / d t=D_{c} \nabla^{2} \tilde{n}_{i}$, ( $\Psi$ represents the trapped ion density with constant quiescent density gradients), while the electron filaments obey $d n_{e} / d t=D_{c} \nabla^{2} \tilde{n}_{\mathbf{i}}-\nu_{e} \tilde{n}_{e}$, where $d / d t \equiv \partial / \partial t+(T c / e B) \mathbf{b} \times \nabla \varphi \cdot \nabla, B$ is the magnetic field, $T$ is the temperature, $e$ is the electron charge, and $c$ is the speed of light. These equations are closed by the quasineutrality condition: $\tilde{n}_{i}-\tilde{n}_{e}=2 n_{0} \varphi$, where $n_{0}$ is the equilibrium density. 
$\varphi$ is the fluctuating electrostatic potential normalized to thermal energy, $\tilde{n}_{i, e}$ is the fluctuating ion (electron) filament dersity, $\nu$ is the collision frequency destroying the fluctuating electron filaments, and $D_{c}$ is a phenomenological dissipation. The $\mathrm{HW}$ and PF are the two major models analyzed in this Letter.

Under the Gaussian-Markovian approximation for the field $\varphi$, the equal-time correlation theory applied to Eq. (1) yields the following set of equations for isotropic turbulence ${ }^{6}$

$$
\begin{gathered}
I_{\psi}(k)=\frac{1}{4 p i k} \int_{0}^{\infty} d r \frac{r^{2} P(r) J_{1}(k r)}{1+\xi / D-P(r)}, \\
P(r) \equiv \frac{2}{r} \cdot \frac{\int_{0}^{\infty} d k k^{2} \Pi(k) J_{1}(k r)}{\int_{0}^{\infty} d k k^{3} \Pi(k)}, \\
D=\pi \int_{0}^{\infty} d k k^{3} \Pi(k)
\end{gathered}
$$

where $\Pi(\mathbf{k}) \equiv \operatorname{Re} \int d \omega G(\omega, \mathbf{k})\left\langle|\varphi(\omega, \mathbf{k})|^{2}\right\rangle$ is the spectral response function, $G(\omega, \mathbf{k}) \equiv$ $-i /(\omega-i \mathbf{k} \cdot \mathbf{D} \cdot \mathbf{k})$ is the one-particle Green's function, $I_{\psi}(k) \equiv|\psi(\mathbf{k})|^{2}$ is the wavenumber spectrum of fluctuating $\Psi, J_{1}$ is the first order Bessel function of the first kind, $D$ is the turbulent diffusion coefficient, and Eq. (2.3) (Dyson-like integral equation) is an expression of diffusive renormalization.

To close the set represented by Eqs. (2), a relationship between $\Pi(k)$ and $I_{\psi}(k)$ is needed. We shall later propose such a closure for PF model. However, for all systems obeying Eqs. (2) extremely interesting and far-reaching results can be obtained by an asymptotic analysis characterized by large $k$ and $\xi \rightarrow 0$. Very generally the total intensity, $W_{\psi} \equiv 2 \pi \int_{0}^{\infty} d k k I_{\psi}(k)$ can be cast [by a direct integration of Eq. (2.1)] in the form

$$
W_{\psi}=-\frac{2}{\left\langle k^{2}\right\rangle} \ln \frac{8 \xi}{D\left\langle k^{2}\right\rangle}+W_{*}
$$

where $\left\langle k^{2}\right\rangle \equiv(\pi / D) \int_{\mathrm{C}}^{\infty} d k k^{5} \pi(k)$ and $W$ stands for the finite part of $W_{\psi}$. Notice that the first term on the right-hand side of Eq. (3) can be (but is no more than) logarithmically: divergent for $\xi \rightarrow 0$ if $\left\langle k^{2}\right\rangle$ remains finite in the same limit. Equation (3) is the first of 
our equations for general asymptotic analysis. By expanding the Fourier-Bessel transform of Eq. (2.1) along with Eq. (2.2) up to $r^{2}$, and equating equal powers, we obtain

$$
\begin{gathered}
D=2 \pi \xi \int_{0}^{\infty} d k k^{3} I_{\psi}(k) \\
D\left\langle k^{2}\right\rangle\left(1+2 \pi \int_{0}^{\infty} d k k^{3} I_{\psi}(k)\right)=2 \pi \xi \int_{0}^{\infty} d k k^{5} I_{\psi}(k),
\end{gathered}
$$

of which Eq. (4.1), a conservation law expressing the saturation condition, can be derived directly from the basic Eq. (1). However, Eq. (4.2) is an intrinsic result of the correlation theory.

The nonlinear relationships between $k$-moments of $\Pi(k)$ and $I_{\psi}(k)$ represented by $\mathrm{Eq}_{1}$ ( 4.2$)$, and by other higher order equations suggest asyrnptotic forms

$$
I_{\psi}(k) \rightarrow \frac{g\left(\xi^{\alpha} k\right)}{k^{d_{\psi}}}, \quad \text { and } \quad \Pi(k) \rightarrow \frac{g\left(\xi^{\alpha} k\right)}{k^{d_{\pi}}}
$$

where $d_{\psi}\left(d_{\Pi}\right)$ denotes the inviscid asymptotic spectral power law for $I_{\psi}(k),[\Pi(k)]$, and $\exists\left(\xi^{\alpha} k\right)(\alpha>0)$ is a factor that provides high $k$ convergence for the $k$ moment integrals. i.e., $\int_{0}^{\infty} d y y^{n} g(y)<\infty[n>-1]$. Equation (3), in conjunction with the definition of $W_{\psi}$. then suggests that $d_{\psi} \geq 2$, with $d_{\psi}=2$ only if $\left\langle k^{2}\right\rangle$ is finite. Substituting Eq. (5) into Eq. (4), it is straightforward to show that $D$ must be finite in the $\xi \rightarrow 0$ limit, and the following relations

$$
\begin{array}{ll}
d_{\Pi}=4+1 / \alpha, d_{\psi}=4-1 / \alpha & \text { if }\left\langle k^{2}\right\rangle \text { is divergent } \\
d_{\psi}=2, \text { and } \alpha=1 / 2 & \text { if }\left\langle k^{2}\right\rangle \text { is finite }
\end{array}
$$

follow. For both of these situations, one still needs one more relation to solve for the indices. It can not come from the correlation theory, because equations from higher $k$ moments do not provide any additional independent relations. It is the very place where the characteristic dynamics of the model enters in a crucial manner.

In the HW model, $|\psi|^{2} \rightarrow\left|k^{2} \varphi\right|^{2}$ for large $k$. This, coupled with the fact that the renormalized propagator ${ }^{7}$ goes as $k^{-2}$ for large $k$, leads to the dynamical relation $d_{\Pi}$ 
$d_{\psi}+6 \geq 8$. With this constraint on $d_{\Pi},\left\langle k^{2}\right\rangle$, must be finite $(\xi \rightarrow 0)$, and a complete solution . for the indices is $d_{\Pi}=8, d_{\psi}=2$ (consistent with -3 inviscid power law of Ref. 2), and $\alpha=1 / 2$. Obviously, in this case, $W_{\psi}$ is logarithmically divergent. This analysis immediately reveals certain inadequacies of the existing literature. For example, a simple minded closure such as $D \approx W_{\psi}^{\gamma}$ assumed in Ref. 6 can be true only if $W_{\psi}$ is finite [ $D$ is finite for all cases]. Another example is related with the question of saturation of $W_{\psi}$ in $\xi \rightarrow 0$ limit. The dissipative term necessary to balance the diffusion for a steady state remains finite even in the zero dissipative limit. This is in contrast to the stated conclusion of Ref. 4. It is evident that a sufficiently large cut-off $k_{\max }\left(>\xi^{-\alpha}\right)$ is crucial for the saturation to be attained.

For the PF, in contrast, $d_{\Pi}-d_{\psi}=2$, because the ion response is essentially adiabatic in the asymptotic regime. The consistent solution for the indices turns out to be $d_{\Pi}=5, d_{\psi}=3$. and $\alpha=1$, implying that $\left\langle k^{2}\right\rangle$ is divergent, while $W_{\psi}$ is finite. It is also interesting to note that the above results for the inviscid power law seem to be insensitive to the special choice of dissipative term at short wavelengths, as long as the model dissipation is so constructed as to form a well-posed problem. For example, if we replace $-\xi \nabla^{2}$ by $\xi \nabla^{4}$, only $\alpha$ is modified $(1 / 3$ for $\mathrm{PF}$, and $1 / 4$ for $\mathrm{HW})$, leaving both $d_{\Pi}$ and $d_{\psi}$ unchanged.

A fully self-consistent solution of the correlation theory requires a closure scheme, i.e., a relation between $\Pi(k)$ and $I_{\psi}(k)$. We now motivate such a scheme for the PF model. The key point is to identify that in this model the inverse decorrelation time in the weak turbulence limit is $\nu_{e}$. Making use of the standard arguments invoked in the diffusive renormalization theories, and adopting the normalizations: $t \rightarrow t / \nu_{e}, r \rightarrow r / k_{0}$ with $k_{0}=\nu_{e} / V_{*}, V_{*} \equiv$ $T c / e B L_{n},\left(L_{n}\right.$ is the density gradient length scale), $D \rightarrow D_{0} D$ with $D_{0} \equiv V_{*}^{2} / \nu_{e}$, and $I_{\psi} \rightarrow 4 I_{\psi} / L_{n}^{2} k_{0}^{4}$, we construct the spectral response function

$$
\Pi(k) \approx \frac{I_{\psi}(k)}{1+D k^{2}}
$$

so designed that the inverse decorrelation time reduces to $\nu_{e}(=1$ in the dimensionless: 
Eq. (7)) in the weak turbulence limit, while it becomes $D k^{2}$ in the strong turbulence limit. With Eq. (7) the set of Eqs. (2) is closed, and simple manipulations lead to a nonlinear differential equation for the "psuedo-correlation function" $P(r)$,

$$
\left(\frac{d}{d r} \frac{1}{r} \frac{d}{d r} r-\frac{1}{D}\right) r P(r)=-\frac{1}{8 D^{2}} \frac{r P(r)}{1+\eta_{0} / D-(r)},
$$

where $\eta_{0} \equiv D_{c} / D_{0}$. From Eq. (2.2) $P(0)=1$, and for small $r$ Eq. (8) gives $P(r)=$ $1-r^{2}\left(1 / 8 \eta_{0}-1\right) / 8 D+\cdots$. The small $r$ behavior of $P$ coupled with the physical requirement that $P(r) \rightarrow 0$ as $r \rightarrow \infty$ defines an eigenvalue problem for $D$, i.e., $D$ is determined as the eigenvalue associated with the well-behaved solutions of the nonlinear Eq. (8). Equation (8) is a consequence of only Eqs. (2.1) and (2.2) implying that the Dyson-like integral equation (2.3), merely provides the additional boundary condition necessary for the nonlinear eigenvalue system. On comparing terms from two equivalent small $r$ expressions of $P(r)$. i.e., from Eq. (2.2) and Eq. (8), we obtain an important formula for the inverse decorrelation time

$$
\tau_{c}^{-1}=D\left\langle k^{2}\right\rangle=\frac{1}{8 \eta_{0}}-1
$$

This equation is precisely a direct consequence of our constructive spectral response function Eq. (7) and the conservation law Eq. (4.1). It suggests that the turbulent diffusion is possible, only if $\eta_{0}$ is less than $1 / 8$, a condition consistent with the linear instability threshold (sufficient for $k \approx 0$, necessary for all $k$ ) for an isotropic spectrum $k^{2} \approx 2 k_{y}^{2}$. It is also interesting to note that the same condition is necessary for the evanescence of $P(r)$ at large $r$; it is for $\eta_{0}<1 / 8$ that the large $r$ behavior is described by the decaying first order modified Bessel function of the second kind ( $K_{1}$-function). Equation (9) also indicates the importance of introducing the $D_{c} \nabla^{2}$ term into the system. Lack of this short, wavelength dissipation results in zero decorrelation time. In fact, for $\eta_{0}=0, d P /\left.d r\right|_{r=0}$ does not exist. and the eigenvalue problem $\left[P(0)=1, d P /\left.d r\right|_{r=0}=0, P(\infty)=0\right]$ associated with Eq. ( $(8)$ is no longer well-posed. 
With a finite $\eta_{0}$ the eigenvalue problem for $P(r)$ can be easily solved by using a shooting code. For a given $P(r)$ the density spectrum $I_{\psi}(k)$ follows from Eq. (2.1), and one can then evaluate $\left\langle k^{2}\right\rangle$, the total turbulence intensity $W_{\psi}$ and other quantities of interests; the problem is fully solved. Numerical results for $D, W_{\psi}$, and $I_{\psi}(k)$ are shown in Figs. 1 and 2 respectively for various values of $\eta_{0}$. The diffusion coefficient seems to be proportional to the intensity, $D\left[\eta_{0}\right] \approx 1.2 W_{\psi}\left[\eta_{0}\right]$, implying that the simple minded closure pertains throughout the entire (from weak to strong) turbulence regime for the PF model.

The strong turbulence regime, characterized by $D k^{2}>\nu_{e}$, also corresponds to $\eta_{0}<1 / 16$. In this regime the dimensionless $D$ is a slowly varying function of $\eta_{0}$ [Fig. 1]. Therefore, the physical $D$ scales as $D_{0}$ and the magnetic field scaling of the diffusion coefficient approaches $1 / B^{2}$, exactly the $B$-scaling of the classical transport theory in contrast to the common belief $^{8}$ that the $B$-scaling for strong turbulence is likely to be Bohm-like, i.e., $D$ should be proportional to $1 / B$. This belief follows from a naive scaling that can be deduced from the diffusive renormalization (Dyson-like) equation, provided the turbulent inverse deccorelation time proportional to $D$ is dominant. Very often, this technique is referred to as estimate of transport by "mixing-length", 8,9 The non-Bohm like diffusion found in the PF model suggests that this estimate may not be universally valid for strong $\mathbf{E} \times \mathbf{B}$ turbulence. Interesting enough, this technique seems to be appropriate for an estimate of higher order $k$-moments. e.g., $\left\langle k^{2}\right\rangle$ in the strong turbulence is found to be consistent with Eqs. (4.1) and (9).

We now resolve a seeming contradiction between our PF result and a simple estimate of diffusion coefficient from the formula for Brownian motion. In this picture $D$ is often estimated by using the stepsize measured by the correlation length, and the frequency of random walk approximated by the characteristic frequency that breaks the coherent motion of waves (the inverse decorrelation time). Consequently, one expects that a spectrum with a peak at low $k$ (long-wavelength condensation) may result in catastrophic diffusion. (Mur solutions for PF clearly show that the density spectra do have peaks at low $k$ whearas the 
energy spectra $w(k) \equiv\left|E_{k}\right|^{2}=k^{2} I_{\psi}(k)$ have peaks at finite $k$ [cf. Fig. 2]. However, in either case the averaged $k^{2}$, which can be approximated by $\left\langle k^{2}\right\rangle$ is not small at all. It can be seen clearly from Fig. 2 that as the turbulence gets stronger, the spectrum (still peaking at low $k$ ) is characterized by a larger $\left\langle k^{2}\right\rangle$, or a shorter correlation length! Therefore, a density spectrum peaking at low $k$ does not necessarily imply a longer correlation length and a catastrophic diffusion.

Using Brownian motion concepts one can derive a quasilinear estimate for the diffusion: $D_{q} \approx\left\langle\gamma_{\mathbf{k}} / k^{2}\right\rangle_{\mathbf{k}}$, where $\langle\cdots\rangle_{\mathbf{k}}$ is the average over $k$. For the PF model, this formula yields $D_{q}=1 / 8-\eta_{0}$, if $k_{y}^{2}$ in $\gamma_{k}$ is assumed to be $k^{2} / 2$ (isotropy). Surprisingly, this formula agrees fairly well with the numerical result from the correlation theory, even in the strong turbulence regime $\left(\eta_{0} \ll 1 / 16\right)$ [cf. Fig.1], where the inverse of decorrelation time is dominated by $D k^{2}$. It appears that as far as the transport is concerned, $\mathrm{PF}$ exhibits three (consistent) quasilinear features: (a) classical $B$-scaling; (b) $D \approx 1.2 W_{\psi}$; and (c) $D \approx D_{q}$. In terms of Brownian motion this interesting behavior can be interpreted as a synchronization of the squared correlation length (stepsize) $\Delta^{2}$ with the decorrelation time $\tau_{c}$. When $\tau_{c}$ behaves as $\eta_{0} / \nu_{e}$ in strong turbulence regime, $\Delta^{2} \approx \eta_{0} / k_{0}^{2}$, so that the resultant diffusion coefficient remains independent of $\eta_{0}$ and near its quasilinear value. Thus, one should not take the above "quasilinear features" to imply that the strong turbulence never ass zrts itself for PF. In fact. neglecting the $D k^{2}$ term in Eq. (2.3) with Eq. (7) leads to poor results except very close to the onset of turbulence [cf. Eq. (4.1)]. While the opposite (large $D K^{2}$ ) approximation leads to Bohm scaling and results quite different from $D\left[\eta_{0}\right] \approx 1.2 W_{\psi}\left[\eta_{0}\right]$. Therefore, the diffusive renormalization (Dyson-like) equation proves to be quite inadequate for transport estimates within the current context; this is in contrast to the scenarios presented in Refs. 8 and 9 .

It is worth pointing out at this stage that the results derived from the correlation theory in this Letter are quite different from those obtained by imposing non-dynamical Ansatzs on turbulence models like PF. ${ }^{10}$ In Ref. 10, a canonical ensemble is assumed for clensity 
fluctuations, resulting in a constant density spectrum in the inviscid range (equivalent to $d_{\psi}=0$ ). Apparently, the argument of Ref. 10 that a divergent density spectrum presumes energy cascading to short wavelengths is, in fact, contrary to our observation deducerl from the correlation theory as illustrated by Eq. (3). In actuality, for diffusive plasmas the total intensity of fluctuating density is not an inviscid constant of motion, although the total density is a Lagrangian inviscid constant of motion. The canonical ensemble Ansatz of Ref. 10 is thus technically unfounded.

The major characteristic of PF-like turbulences is the shrinkage of the correlation lengths for short wavelength dissipation. This characteristic, independent of details and merely pertaining to the dynamical nature embodied in $d_{\Pi}-d_{\psi}<4$, implies a breakdown of "mixinglength" estimates for the turbulence level. The physical total intensity in strong turbulence regime is proportional to $1 / k_{0}^{2} \approx \Delta^{2} / \eta_{0}$, rather than to $\Delta^{2}$ (the squared correlation length) alone. Breakdown of "mixing length" has been recently reported on experiments in the supershot regime on the TFTR tokamak. ${ }^{11}$ The above analysis then suggests that the corresponding turbulence in the supershot regime on TFTR may be of a PF-like genre, and that the improved confinement in the supershot regime may be interpreted as achieving the type of turbulence whose transport is characterized by shrinking correlation lengths.

In conclusion, the introduction of an appropriate short wavelength dissipation is found to be crucial for formulating a well-posed problem within the framework of equal-time correlation theory to investigate the dynamical nature of $2 \mathrm{D}$ plasma turbulence with one Lagrangian inviscid constant of motion. For a two-field problem the inviscid power law and the degrees of divergence of various $k$-moments are characterized by three power indices, $d_{\Pi}, d_{\psi}$, and $\alpha$. By the correlation theory for Lagrangian inviscid constant of motion only two independent relations can be obtained. Therefore, the full determination of the indices relies on the dynamical nature of the system, which could yield, for instance the value $d_{\Pi}-d_{\psi}$. This general scheme provides a powerful approach to solve the inviscid power law $\left(d_{\psi}\right)$, and hat 
been applied to $\mathrm{HW}$ and PF to illustrate the relationship between the inviscid power law and the characteristics of transport. For HW $\left(d_{\psi}=2\right) W_{\psi}$ is logarithmically divergent, and independent of $\alpha$, while $\left\langle k^{2}\right\rangle$ is finite. For PF $\left(d_{\psi}=3>2\right)$, on the other hand, $W_{\psi}$ is finite (clump theory ${ }^{12}$ fails), while $\left\langle k^{2}\right\rangle$ is algebrically divergent with the degree depending upon $\alpha$. and the dissipation model. In both cases the diffusion coefficient $D$ is shown to be finite in the inviscid limit. In a sense the 2D turbulences, on the basis of the divergence involved, can be catagorized into HW-like and PF-like. In particular for PF, we constructed a physically reasonable spectral response function on basis of the above asymptotic analysis to solve selfconsistently for the spectrum and the transport. The solution exhibits several "quasilinear features," which seems to stem from a synchronization of the squared correlation length with the decorrelation time in strong turbulence regime. The shrinkage of the correlation length is a direct consequence of the divergent nature of $\left\langle k^{2}\right\rangle$, and not an artifact of the particular choice [Eq. (7)] of the spectral response function. For turbulences with this synchronization (PF-like) "mixing-length" breaks down, so that the diffusive renormalization (Dyson-like) equation fails to provide a simple tool for the estimate of transport; the quasilinear formulas, on the other hand, yield much better estimates. This theory combined with the recent experimantal observation ${ }^{11}$ suggests an interpretation for the improved confinement in the supershot regime on the TFTR tokamak.

\title{
DISCLAIMER
}

\begin{abstract}
This report was prepared as an account of work sponsored by an agency of the United States Government. Neither the United States Gnvernment nor any agency thereof, nor any of their employees, makes any warranty, express or implied, or assumes any legal liability or responsibility for the accuracy, completeness, or usefulness of any information, apparatus, product, or process disclosed, or represents that its use would not infringe privately owned rights. Reference herein to any specific commercial product, process, or service by trade name, trademark, manufacturer, or otherwise does not necessarily constitute or imply its endorsement, recommendation, or favoring by the United States Government or any agency thereof. The views and opinions of authors expressed herein do not necessarily state or reflect those of the United States Government or any agency thereof.
\end{abstract}




\section{References}

${ }^{1}$ A.N. Kolmogorov, C.R. Acad. Sci, USSR 30, 301 (1941); U. Frisch, Phys. Ser. 9, 131 (1985).

${ }^{2}$ R.H. Kraichnan, Phys. Fluids 10, 1417 (1967).

${ }^{3}$ A. Hasegawa and K. Mima, Phys. Fluids 21, 87 (1978); D. Fefy and D. Montogomery, Phys. Fluids 22, 246 (1979); A. Hasegawa, Y. Kodama, and C.Maclennan, Phys. Fluids 22, $2122(1979)$.

${ }^{4}$ M. Wakatani and A. Hasegawa, Phys. Fluids 27, 611 (1984).

${ }^{5}$ B.B. Kadomtsev and O.P. Pogutse, Sov. Phys. JETP 24, 1172 (1967).

${ }^{6}$ Y.Z. Zhang and S.M. Mahajan, Phys. Fluids B 5, 2000 (1993).

${ }^{7}$ There are too numeral references on this issue. We refer to the review paper by J. Krommes in "Handbook of Plasma Physics," ed. M.N. Rosenbluth, et al.

${ }^{8}$ J.D. Callen, Phys. Fluids B 4, 2142 (1992).

${ }^{9}$ J.D. Callen, B.A. Carreras, and R.D. Stambough, Physics Today, p. 34, January (1992).

${ }^{10}$ P.H. Diamond, and H.Biglari, Phys. Rev. Lett. 65, 2865 (1990).

${ }^{11}$ E. Mazzucato and R. Nazikian, Phys. Rev. Lett. 71, 1840 (1993); Bull. Am. Phys. Soc. 38, 1906 (1993).

${ }^{12}$ T.H. Dupree, Phys. Fluids 15, 334 (1972). 


\section{Figure Captions}

1. The diffusion coefficient, $D$, (hollow circles) and the total turbulence intensity $W_{v}$ (dark circles) versus $\eta_{0}$ from the numerical solutions of Eq. (8). The solid curve is the quasilinear formula $D_{q}=1 / 8-\eta_{0}$.

2. The spectra $I_{\psi}(k)$ (solid curves: $a 1, b 1, c 1$ ) and wave energy spectra $w(k) \equiv k^{2} I_{\psi}(k)$ (dashed curves: $a 2, b 2, c 2$ ). The curves $a, b$ and $c$ correspond to $\eta_{0}=0.1,0.01$ and 0.001 respectively. 


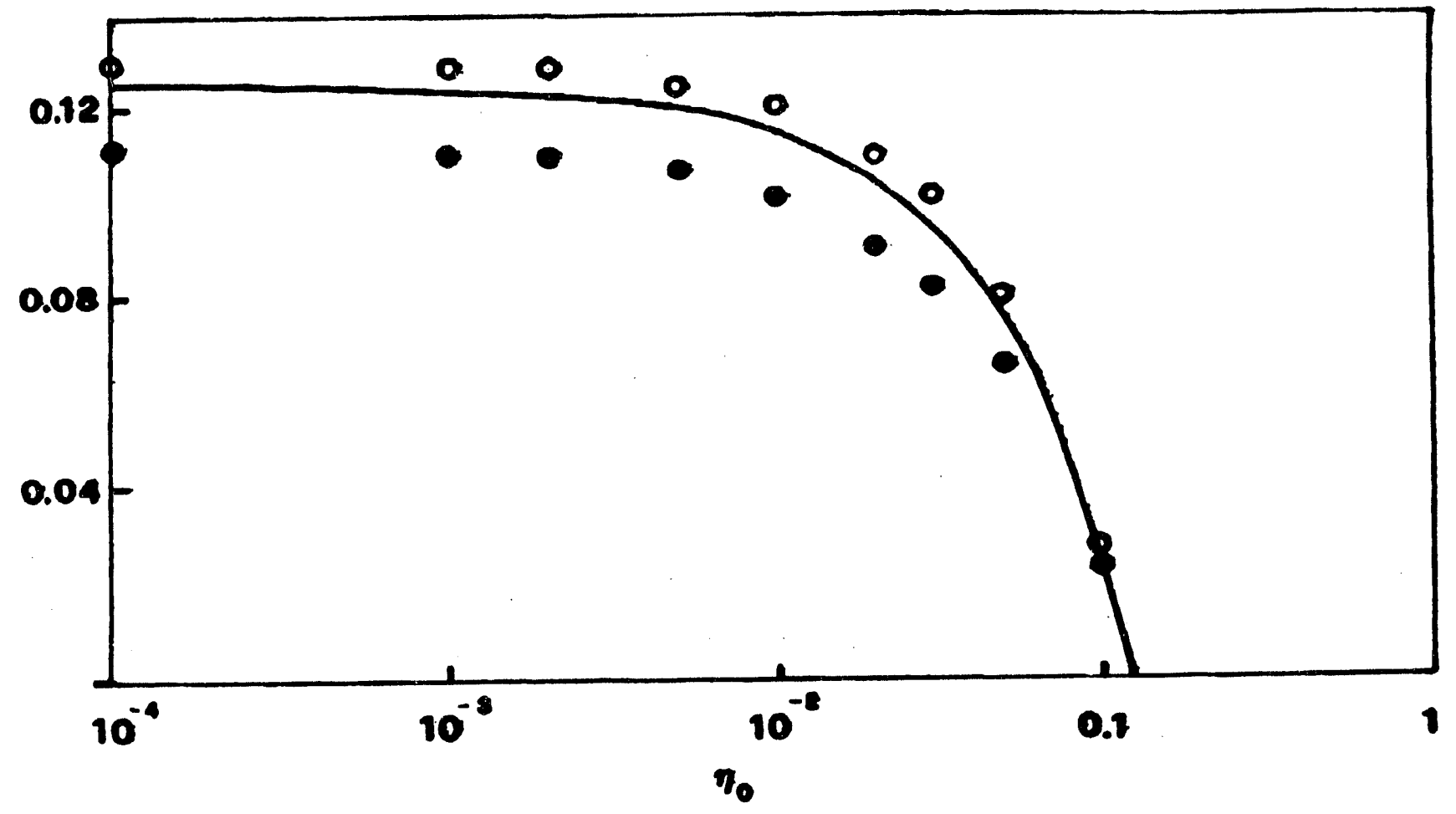

T18.1 


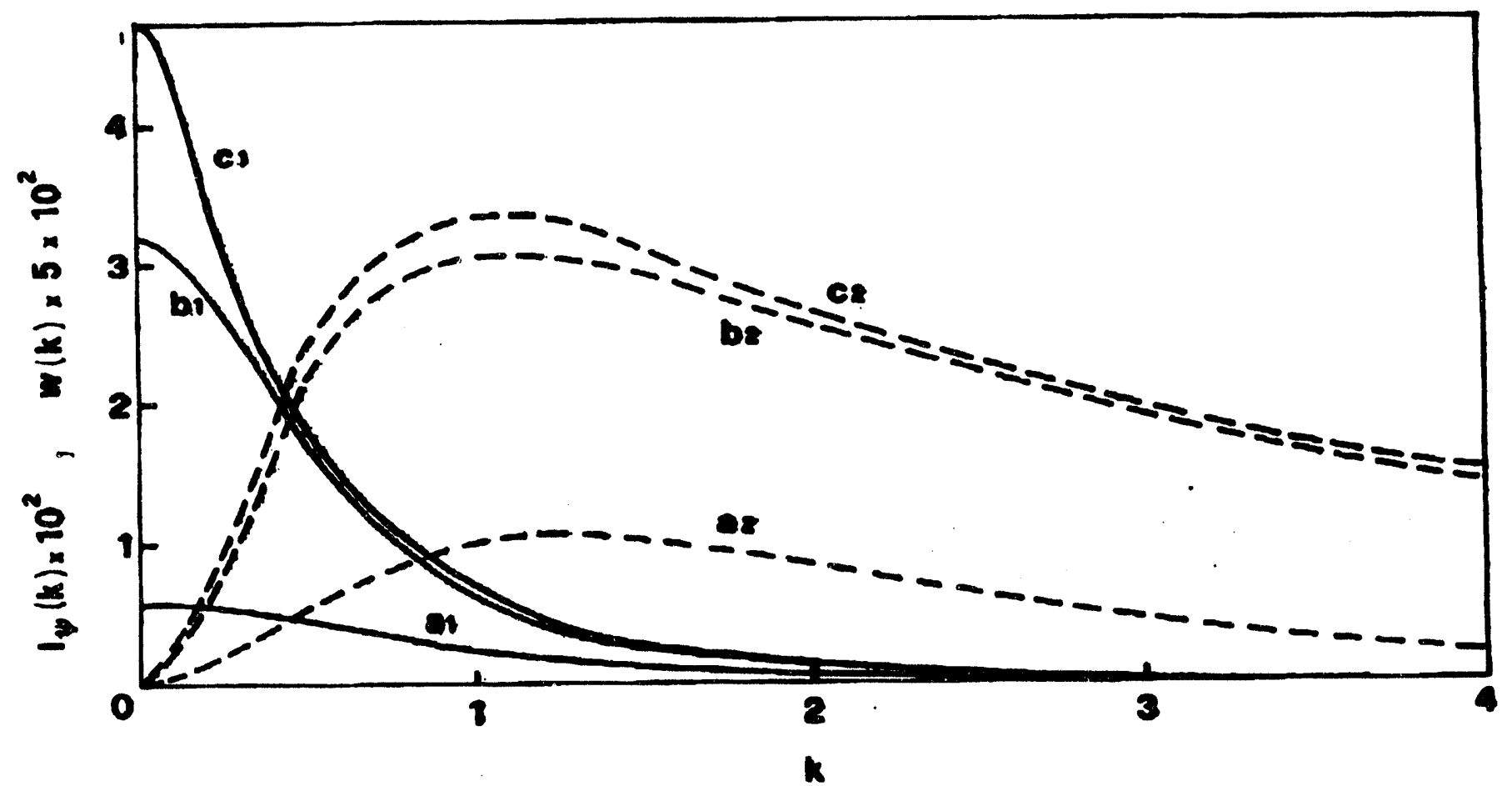

718.2 

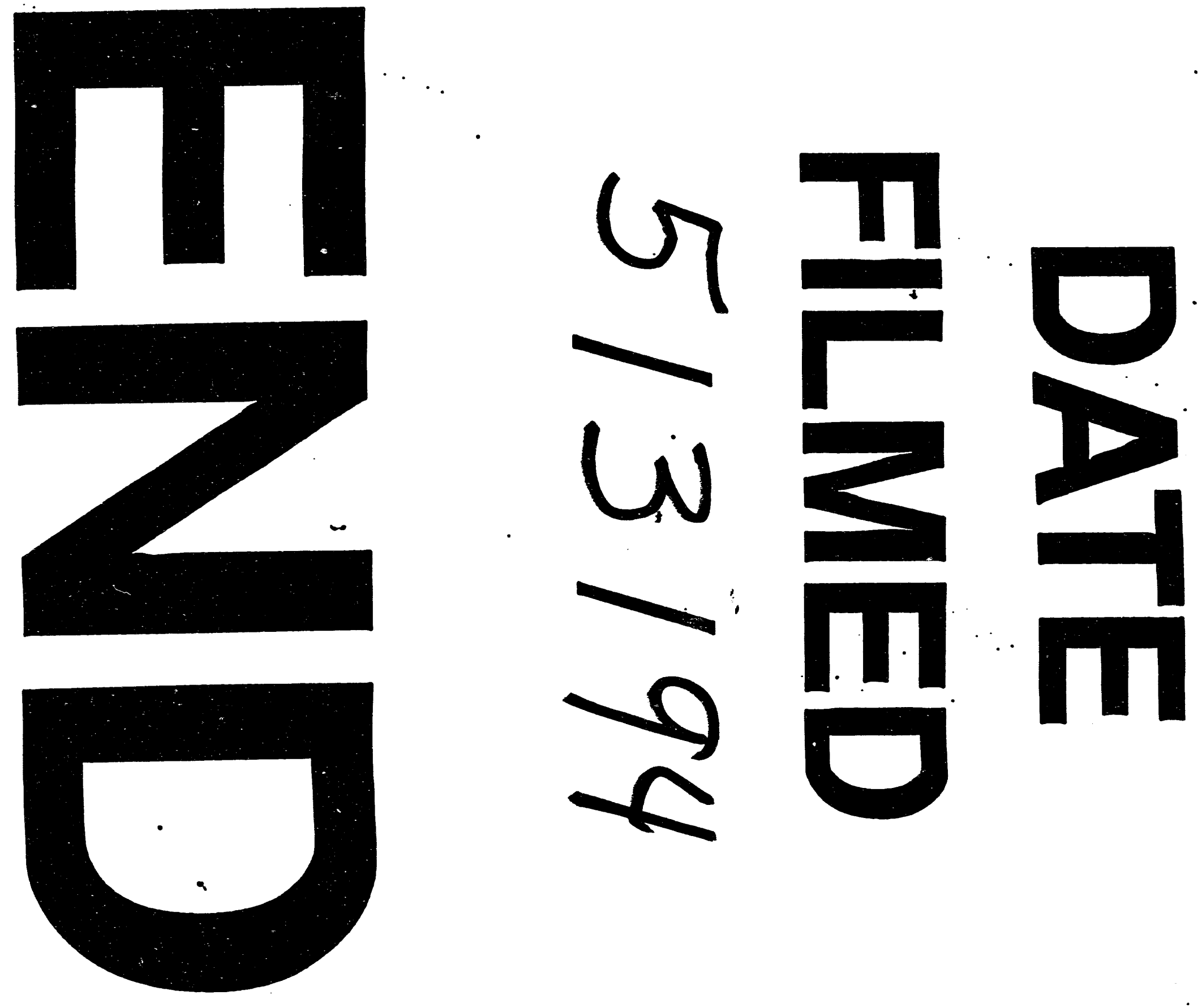
